\title{
Use of antikeratin antibodies to distinguish between rheumatoid arthritis and polyarthritis associated with hepatitis $\mathbf{C}$ infection
}

\author{
A. Kessel, I. Rosner, E. Zuckerman, T.D. Golan, E. Toubi \\ Division of Clinical Immunology and Allergy, Bnai Zion Medical Center and Faculty of Medicine, Technion, Haifa, Israel
}

\begin{abstract}
SUMMARY
Objective: To investigate whether antikeratin antibodies (AKA) could be usefull in the differential diagnosis of patients with rheumatoid arthritis (RA) compared to patients with hepatitis $C$ virus $(H C V)$ associated polyarthritis, who are seropositive for rheumatoid factor $(R F)$.

Methods: AKA were assayed in 3 different groups of patients; all were RF seropositive: Group 1: 25 patients with HCV associated polyarthralgia or arthritis. Group 2: 33 patients with RA. Group 3: 13 patients with autoimmune disorders other than RA. Fifteen healthy individuals served as controls.

Results: AKA were detected in 20/33 patients with RA (60.6\%) compared to only 2/25 patients (8\%) with HCV associated arthritis $(p<0.0001)$. AKA were observed in $2 / 13$ patients of Group 3 (15.3\%). These results were also statistically different from those of patients with RA $(p=0.008)$. AKA were not found in the sera of the healthy controls. Conclusion: AKA is a useful marker to differentiate patients with RA from those with hepatitis $C$ arthritis.
\end{abstract}

Reumatismo, 2001; 53(2):156-157

\section{COMMENTO}

9 infezione da HCV rappresenta oggi un problema per molti specialisti di differente estrazione. Tra questi particolarmente coinvolti sono i reumatologi. Se volessimo infatti arbitrariamente elencare le problematiche che l'infezione da HCV pone al clinico nella pratica quotidiana, potremmo distinguerle in problematiche cliniche e problematiche terapeutiche.

Tra le prime sicuramente importanti sono le manifestazioni extraepatiche che il virus può causare, molte di queste quali la crioglobulinemia mista tipo II e la scialoadenite linfocitaria cronica sono ormai ben definite altre, quali l'interstiziopatia polmonare e il liken ruber planus e altre ancora sono tutte da verificare. Esiste inoltre il frequente problema della diagnosi differenziale tra patologie primitive e patologie secondarie all'infezione, del tutto simili tra loro, che sono difficili da distinguere soprattutto quando le manifestazioni cliniche sono al loro esordio.

Nel secondo gruppo di problematiche, non meno importanti delle prime e con queste direttamente correlate, segnaliamo i problemi legati alle terapie. In primo luogo c'è la scelta terapeutica in sindromi reumatiche sostenute dal virus che mimano altre patologie autoimmuni o immunomediate primitive. Altro punto caldo è quello della scelta dei farmaci da utilizzare nel trattamento di patologie autoimmuni o immunomediate in presenza di una infezione da HCV, parlo soprattutto dei FANS, dei corticosteroidi dei farmaci di fondo, degli immunosoppressori e, non ultimo, delle conseguenze che possono avere sulla storia naturale dell'infezione nuovi farmaci quali quelli biologici (Remicade, Infliximab) entrati di recente nell' armamentario terapeutico del reumatologo. Ultimo aspetto terapeutico e quello delle conseguenze che può avere l'uso degli interferoni su patologie autoimmuni preesistenti ovvero sul ruolo che essi possono avere nella comparsa di patologie autoimmuni ex novo. È infatti acquisito da tempo il fatto che possa risultare molto più rischioso trattare una patologia autoimmnune con interferoni, quale l'epatite cronica tipo I, piuttosto che trattare con corticosteroidi patologie sostenute dall'infezione da HCV quale l'epatite cronica da HCV. 
Tutte queste tematiche potrebbero essere tema di un editoriale mirato perché di frequente riscontro quotidiano considerando che la prevalenza dell'infezione da $\mathrm{HCV}$, sintomatica e non, nella popolazione generale, viene calcolata oggi intorno al 3\% con punte più alte in alcune popolazioni anche in Italia. L'articolo che segnaliamo oggi nella nostra rubrica affronta uno dei problemi sopra citati e ne propone una possibile soluzione, problema che assume anche grande importanza per i riflessi terapeutici che questo comporta, ci riferiamo alla diagnosi differenziale tra artrite reumatoide e poliartrite similreumatoide direttamente sostenuta dal virus dell'epatite C. Gli autori, avendo selezionato tre gruppi di pazienti due dei quali rispettivamente con poliartite o poliartralgie e con artrite reumatoide e uno con altre malattie autoimmunitarie, tutti con in comune la positività del fattore reumatoide, hanno concluso che il riscontro degli anticorpi circolanti anti-cheratina possa assumere un valore di grande importanza nella diagnosi differenziale tra AR e artrite da HCV. Se questo dato fosse confermato sarebbe risolto in parte uno dei problemi di non poca importanza clinica e terapeutica che il reumatologo deve spesso affrontare nella pratica quotidiana.

Mauro Galeazzi Istituto di Reumatologia, Siena 